\title{
Epidemiologic surveillance of nosocomial infections in a Pediatric Intensive Care Unit of a developing country
}

\author{
María R Becerra ${ }^{1}$, José A Tantaleán ${ }^{2 *}$, Víctor J Suárez ${ }^{3}$, Margarita C Alvarado ${ }^{4}$, Jorge L Candela ${ }^{5}$, Flor C Urcia ${ }^{6}$
}

\begin{abstract}
Background: Nosocomial Infections (NI) are a frequent and relevant problem. The purpose of this study was to determine the epidemiology of the three most common NI in a Pediatric Intensive Care Unit from a developing country.

Methods: We performed a prospective study in a single Pediatric Intensive Care Unit during 12 months. Children were assessed for $3 \mathrm{NI}$ : bloodstream infections (BSI), ventilator-associated pneumonia (VAP) and urinary tract infections (UTI), according to Center for Disease Control criteria. Use of devices (endotracheal tube [ETT], central venous catheter $[\mathrm{CVC}]$ and urinary catheter [UC]) was recorded.

Results: Four hundred fourteen patients were admitted; 81 patients (19.5\%) developed $85 \mathrm{NIs}$. Density of incidence of BSI, VAP and UTI was 18.1, 7.9 and 5.1/1000 days of use of CVC, ETT and UC respectively. BSI was more common in children with CVCs than in those without CVCs (20\% vs. 4.7\%, p < 0.05). Candida spp. was the commonest microorganism in BSI (41\%), followed by Coagulase-negative Staphylococcus (17\%). Pseudomonas (52\%) was the most common germ for VAP and Candida (71\%) for UTI. The presence of NI was associated with increased mortality (38.2\% vs. $20.4 \%$ in children without NI; $p<0.001$ ) and the median length of ICU stay ( 23 vs. 6 days in children without $\mathrm{NI} ; \mathrm{p}<0.001)$. Children with $\mathrm{NI}$ had longer average hospital stay previous to diagnosis of this condition (12.3 vs. 6 days; $p<0.001)$.
\end{abstract}

Conclusions: One of every 5 children acquires an $\mathrm{NI}$ in the PICU. Its presence was associated with increased mortality and length of stay. At the same time a longer stay was associated with an increased risk of developing $\mathrm{NI}$.

\section{Background}

Nosocomial Infections (NIs) are a frequent problem, particularly in Intensive Care Units (ICU). In Europe, incidences range from $1 \%$ in General Pediatric wards up to $23.6 \%$ in Pediatric ICUs (PICU) [1]. PICU studies report incidences between $6.1-15.1 \%$ [2,3] whilst a cross-sectional study found a prevalence of $11.9 \%$ [4].

Bloodstream infections (BSI) are the most common NI in PICUs (28-52\% of all) [2-6]; pneumonia (including ventilator associated pneumonia, VAP) and urinary tract infection (UTI), followed by enteric, surgical site and skin [1-4]. BSI and pneumonia are responsible for

\footnotetext{
* Correspondence: tantaleanjose@hotmail.com

${ }^{2}$ Pediatric Intensivist, Master in Medicine, Instituto Nacional de Salud del

Niño and Universidad Nacional Federico Villarreal, Lima, Peru

Full list of author information is available at the end of the article
}

approximately $50 \%$ of NIs, and UTI causes an additional $12-22 \%[3,7]$. Although VAP is the most frequent NI in adults $(8-28 \%$ of adults under mechanical ventilation, MV, developed VAP [8]), pediatric studies report incidences of 2 - 17\% [9-12]. Only two pediatric studies have found VAP to be the most frequent NI in PICU $[1,13]$.

All aforementioned studies, except two [12,13], come from developed countries. In contrast to PICUs from developed countries, those in developing countries often admit more critically ill patients, with medical conditions rather than surgical, and with lower ages and socioeconomic level [14]. A study from Brazil found that yeasts and Gram negative bacteria were the most frequent isolates in blood cultures [13]. Another study in adults from developing countries found greater frequency of NI compared to ICUs in the USA, despite similar device use 
rates [15]. So, it is possible that there could be differences in the demographic and epidemiologic characteristics of NI in PICUs from developing countries. The objective of this study was to understand the epidemiological profile of the 3 most common NIs (BSI, VAP and UTI) in a PICU of a developing country.

\section{Methods}

A prospective study, which followed rules of active epidemiologic surveillance, was performed in the PICU at the Instituto Nacional de Salud del Niño (INSN) of Lima, Peru. This is a national reference center admitting patients from all over the country. It has 16 beds for children between 0 and 18 years, admitting around 500 patients per year. Cardiac surgery and burn patients have a dedicated ICU. Usually no preterm infants are admitted to the PICU, and no prophylactic antibiotic or antifungal therapy is given for high risk newborns. Most admissions come from the Emergency Department.

This study, approved by the Committees on Ethics of the INSN and the Instituto Nacional de Salud (INS) of Lima occurred over a twelve month period (June 21st 2006 through to June 20th 2007).

The CDC diagnostic criteria [16] were used, along with those of the International Pediatric Sepsis Consensus Conference [17], as was recently proposed [18]. An infection was defined as nosocomial when it appeared after 48 hours of PICU admission and if there was no evidence that the infection was present or incubating at the time of PICU admission.

Diagnosis of BSI included both laboratory confirmed infections, and clinical sepsis. Clinical sepsis was defined as such when a physician prescribed treatment for sepsis, in a patient with signs and symptoms of sepsis with no other apparent site of infection, and blood culture was negative or no blood culture was performed. Laboratory confirmed infection was diagnosed when: a) a recognized pathogen was isolated from one or more blood cultures and this pathogen was not associated to infection at another site in a patient with signs or symptoms of severe infection or; b) a patient had a diagnosis of sepsis and a common skin contaminant (like coagulase-negative Staphylococcus, CNS) was isolated from two or more blood cultures drawn on separate occasions, or when it was isolated in a patient with an intravascular line and had treatment for sepsis prescribed by a physician. BSI was diagnosed in both, children with and without CVC. Blood samples were obtained from a separate peripheral venipuncture after skin preparation with iodine solution, in amounts according to patient's age (<1y: $1-2 \mathrm{ml} ; 1-12 \mathrm{y}: 1-10 \mathrm{ml} ;>12 \mathrm{y}: 10 \mathrm{ml})$.

VAP was defined as early when it developed during the first 4 days under $M V$, and late VAP when it developed after the fifth day [19]. Tracheobronchial samples were collected through deep tracheal aspirates from endotracheal tubes (ETT) with a closed suction technique and urine samples were aseptically aspirated from the sampling port of the urinary catheter (UC). No invasive technique for sampling of tracheal secretions was used.

UTI was classified as symptomatic UTI, asymptomatic bacteriuria and other UTIs, as defined by CDC criteria [16]. To consider urine samples as positive the number of colony forming units $(\mathrm{CFU} / \mathrm{ml})$ had to be greater than $10^{5} \mathrm{CFU} / \mathrm{ml}$.

All samples (blood, tracheal and urine) were obtained only after an infection was suspected. All samples were collected in containers with antibiotic remover (BACTEC, Becton Dickinson, Microbiology system, Cockeysville, MD, USA) and processed in the INSN Laboratory. Some isolated bacteria were sent to the INS to confirm bacterial susceptibility by disk diffusion method. All yeasts isolated at the INSN were sent to the INS and antifungal susceptibility was determined by disk diffusion method.

Other NIs such as upper respiratory, surgical, enteric, skin or infections at other sites, were not surveyed. All admitted children were assessed daily (Monday to Saturday) by one of the authors (MRB, MCA or JAT) who ascertained the presence of signs compatible with NI and use of devices (CVC, ETT or UC) up to 48 hours of discharge from the PICU.

At the time of the study there were no approved rules for insertion of CVCs, but for all insertions masks, sterile gowns and gloves were used, cleaning the skin with clorhexidine $2 \%$ plus disinfection with alcohol $70 \%$ and covering the puncture site with sterile gauze and transparent dressing. Written rules for prevention of catheter-associated BSI approved by the Infection Control Committee were in use since 2003. The connecting tubes were changed every third day, unless CVCs were used for TPN or blood transfusions, in those cases it was changed every day. Any drug administration was preceded by cleansing of the three-way stopcock with $70 \%$ alcohol. The puncture site was cleansed with alcohol only if soiling was observed. There were written guides for ETT and UC care. Aspiration of secretions was made according to patient's condition, with aseptic technique, previously irrigating with sterile normal saline. All patients with UC in place had a closed drainage system, except infants under 1 month of age. Manipulation of UC was always with aseptic technique.

The total of patient-days and days of use of devices (CVC, ETT and UC) were recorded. NIs can be described by the incidence rate (per 100) using the number of each assessed infection as numerator and the number of patients with risk factors as denominator, or by device-associated incidence rate (DI) (per 1000) 
dividing the number of each assessed infection by the number of days which patients were exposed to risk factors. For calculating density of incidence of BSI and UTI only children with CVC or UC were considered. A ventilator, CVC or UC day was considered as such only if the device was placed during the morning hours (until 13:00). The ratio of use of devices was estimated dividing number of days of use of a device between days the patients spent at the ICU.

Crude excess mortality was defined as the difference between mortality in children with and without NI. PRISM score was calculated during the first 24 hours of admission.

We performed descriptive analysis of central tendency and variability measurements as well as rate estimates. To perform bivariate and multivariate analysis to determine the risk for NI linear generalized models with Poisson family and log link adjusted values for age, sex and PO condition were used. Furthermore, the Kaplan-Meier analysis for survival was performed for the time of the NI event at the ICU and for death at ICU. Categorical variables were evaluated using the chi square or Fisher' exact test, as appropriate. Continuous variables were evaluated using the Mann-Whitney test. Proportions were compared using the $\mathrm{z}$ test, median test to compare medians and Mann Whitney's U test to compare averages. We used the stata 8.0 statistical program (Single user Stata for Windows perpetual license. Serial number: 8199049125. Licensed to: Instituto Nacional de Salud).

\section{Results}

\section{Demographic features and use of devices}

There were 444 admissions for 414 patients (18 patients were admitted twice and 6 were admitted 3 times). The average age was 52.8 months (range: 0.02 - 214; SD 62.9; first quartile: 4.0, third quartile: 103.7) and the median was 19 months; 189 children (46\%) were under 1 year. Demographic features, reason for admission, comorbidities, patient-days, use of devices, length of stay and mortality for patients with and without NI are described in Table 1. The group of children with NI was younger; however reasons for admission to PICU as well as comorbidities were similar in both groups. No preterm infant was admitted during the study period.

Table 1 Demographic features, comorbidities and device use in children

Of all admissions, 52\% came from the Emergency Department. Of admitted children, $43 \%$ had a CVC, $70 \%$ ETT and 38\% UC. The ratios of use of devices were $0.46,0.74$ and 0.25 for CVC, ETT and UC respectively. PICU mortality for the entire population during the study period was $23.6 \%$. The Kaplan-Meier analysis
Table 1 Demographic features, comorbidities and device use in children

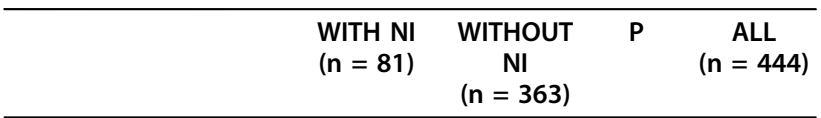

\begin{tabular}{|c|c|c|c|c|}
\hline AGE & & & & \\
\hline months, median & 7.7 & 24 & 0.002 & 19 \\
\hline \multicolumn{5}{|l|}{ GENDER } \\
\hline$M / F$ & $50 / 31$ & 192/171 & - & $242 / 202$ \\
\hline Medical n (\%) & $68(84)$ & $272(75)$ & 0.053 & 340 (76.6) \\
\hline respiratory & 21 & 60 & - & 81 \\
\hline neurological & 8 & 69 & - & 77 \\
\hline septic & 21 & 49 & - & 70 \\
\hline others & 18 & 94 & - & 112 \\
\hline Post operative n (\%) & $13(16)$ & $91(25)$ & 0.053 & $104(23.4)$ \\
\hline neurosurgery & 2 & 45 & - & 47 \\
\hline others & 11 & 46 & - & 57 \\
\hline \multicolumn{5}{|l|}{ COMORBIDITY } \\
\hline total (\%) & $45(56)$ & $163(45)$ & 0.081 & $208(47)$ \\
\hline $\begin{array}{l}\text { congenital } \\
\text { syndromes }\end{array}$ & 30 & 79 & - & 109 \\
\hline chronic conditions & 11 & 52 & - & 63 \\
\hline neoplasia & 2 & 26 & - & 28 \\
\hline others & 2 & 6 & - & 8 \\
\hline PRISM * average & $7 \pm 6.7$ & $6.47 \pm 6.63$ & 0.74 & \\
\hline PATIENT DAYS & 2689 & 1914 & - & 4603 \\
\hline
\end{tabular}

\begin{tabular}{|c|c|c|c|c|}
\hline DEVICE USE & & & & \\
\hline CVC days & 1180 & 922 & - & 2102 \\
\hline ventilator days & 2091 & 1329 & - & 3420 \\
\hline UC days & 620 & 550 & - & 1170 \\
\hline \multicolumn{5}{|l|}{$\begin{array}{l}\text { DEVICE UTILIZATION } \\
\text { RATIO }\end{array}$} \\
\hline CVC & 0.44 & 0.48 & - & 0.46 \\
\hline$E T T$ & 0.78 & 0.69 & - & 0.74 \\
\hline UC & 0.23 & 0.28 & - & 0.25 \\
\hline ICU STAY (days)median & 23 & 6 & $\begin{array}{c}< \\
0.001\end{array}$ & 6.9 \\
\hline \multicolumn{5}{|l|}{ MORTALITY } \\
\hline PICU, n (\%) & $31(38.3)$ & $74(20.4)$ & $\begin{array}{c}< \\
0.001\end{array}$ & 105 (23.6) \\
\hline Hospital, $28 d(\%)$ & $36(44.4)$ & 119 (32.7) & 0.060 & 155 (34.9) \\
\hline
\end{tabular}

* data from 97 patients (20 with and 77 without $\mathrm{NI}$ ).

$\mathrm{NI}$ : nosocomial infection; CVC: central venous catheter; ETT: endotracheal tube; UC: urinary catheter.

shows that $50 \%$ of deaths occurred by the sixth week at PICU (Figure 1).

\section{Figure 1. Time of death}

\section{Features of Nosocomial Infections (Table 2)}

Out of 414 admitted patients 81 (19.5\%) developed an NI, most of them in children $<1$ year $(56.5 \%$ of NIs) 


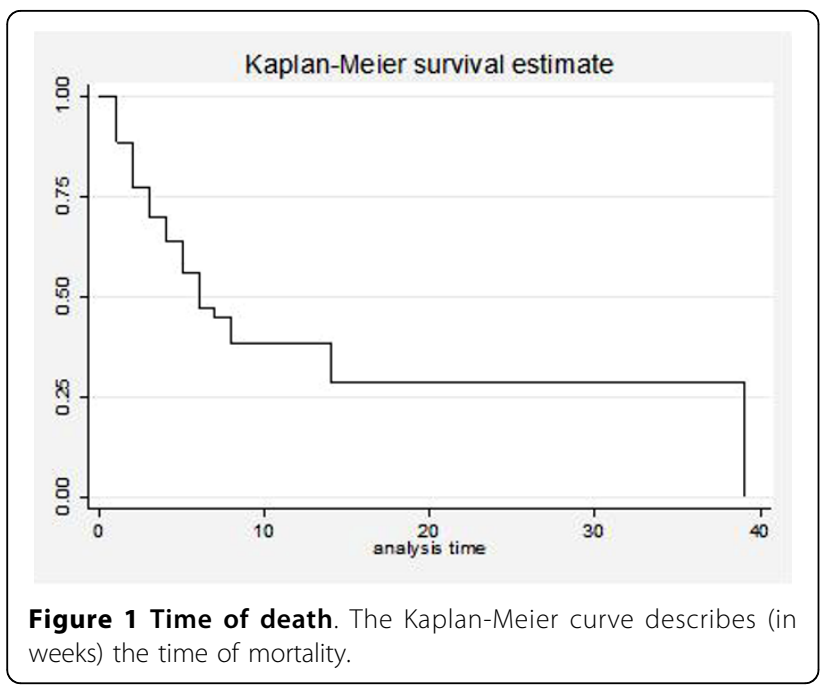

and in males (50/31). The most common NI was BSI (49 cases), 29 of them with laboratory confirmation and 20 with clinical sepsis; 38/49 BSIs presented in children with CVC; amongst these 38 catheter-related BSI some microorganism was isolated in 29 , and 9 were clinical sepsis. VAP was observed in 27 cases (5 early; 22 late VAP) and symptomatic UTI in 9; 6/9 UTIs presented in children with UC. DI for BSI, VAP and UTI was 18.1, 7.9 and 5.1 per 1000 days of use of CVC, MV and UC respectively. Incidence rate for $\mathrm{NI}$ in children with $\mathrm{CVC}$, ETT or UC was 3.0, 2.7 and 2.7 per 100 patient days, respectively. Median ICU stay before NI diagnosis was 7 days.

\section{Table 2. Features of nosocomial infections}

Rates of incidence were: 38/190 (20\%) for BSI; 27/313 (8.6\%) for VAP and 6/168 (3.6\%) for UTI. Rate of incidence of BSI in patients with CVC was greater than in patients without CVC (20\% vs. $4.7 \%, \mathrm{p}<0.05)$, whilst rate of incidence of UTI in patients with UC was not significantly greater than in patients without UC $(3.6 \%$ vs. $1.1 \%, \mathrm{p}=0.14$ ).

The median length of stay (LOS) in ICU for children without NI was 6 days (range 2-60), whilst in those with NI it was 23 days (range $5-268)(p<0.001)$. The LOS, previous to diagnosis of NI, in children with NI was significantly greater than LOS in children without NI

Table 2 Features of nosocomial infections

\begin{tabular}{lc}
\hline Infections acquired at ICU & Rate \\
\hline Rate of infection (UCI)/1000 & 26,3 \\
\hline Ventilator Associated Pneumonia rate/1000 & 7,9 \\
\hline Catheter Related Blood Stream Infection rate/100 & 18,1 \\
\hline Catheter Related Urinary Infection rate/100 & 5,1 \\
\hline Calculated device-associated infection rate/1000 & 12,5 \\
\hline
\end{tabular}

(12.3 vs. 6 days, $\mathrm{p}<0.001)$. The average additional stay after diagnosis of NI was 20.8 days, which accounted for $60 \%$ of stay for infected patients. Median LOS for individual NI was 22.5, 25 and 34 days for BSI, VAP and UTI, respectively.

PRISM was calculated in only $22 \%$ of patients and it was no different between those patients with NI (7) compared to those without NI (6.6) $(\mathrm{p}=0.74)$.

Mortality in PICU was greater amongst patients with NI (38.3\%) than in patients without NI $(20.4 \%)$ ( $\mathrm{p}<$ 0.001 ), with a crude excess mortality of $17.8 \%$. Mortality after 28 days of discharge from PICU was greater in children with NI, although without statistical significance $(44.4 \%$ vs. $32.7 \%$; $\mathrm{p}=0.06)$. Mortality for BSI, VAP and UTI was $30.6 \%, 51.8 \%$ and $22.2 \%$ respectively. Mortality in children with CVC and BSI was greater than in children with CVC but no BSI, with no statistical significance $(42.1 \%$ vs. $28.9 \%, \mathrm{p}=0.135)$. Mortality in patients with VAP was greater than in patients without VAP $(51.8 \%$ vs. $31.1 \%, \mathrm{p}<0.05)$. Mortality in patients with VAP was 59\% (late) and 20\% (early). Mortality for UTI (22.2\%) was not different from that of the entire population $(23.6 \%)$.

\section{Distribution of pathogens \\ BSI (29 isolates)}

In $59 \%$ of cases of BSI at least one microorganism was isolated in blood cultures. The most common was Candida (41\% of isolations) of which $10(83 \%)$ were nonalbicans (two cases of glabrata, krusei, tropicalis each; one case of lipolitica, guilliermodi, parapsilosis, sp each) and 2 (17\%) were $C$ albicans. Candida was only isolated from children with CVC. Enterococcus was not isolated. Figure 2 shows pathogens isolated in BSI. Out of 4 isolated Pseudomonas, 3 were aeruginosa and one fluorescens; included in "others" were the following: Klebsiella oxytoca, Acinetobacter sp, Providencia stuartii, Acremonium $s p$ and Kodamaea ohmeris (one each).

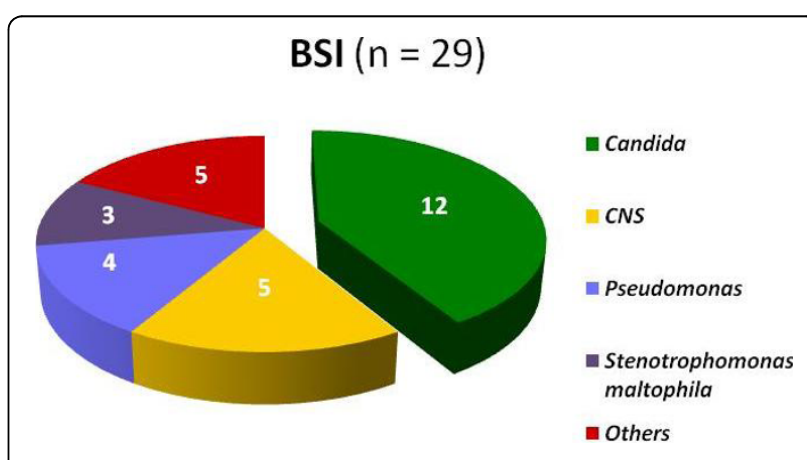

Figure 2 Pathogens isolated in ICU patients with BSI. Candida spp was the most common microorganism in bloodstream infection. 
Figure 2. Pathogens isolated in ICU patients with BSI VAP (31 isolates)

In $97 \%$ of VAPs at least one microorganism was isolated from tracheal aspirate, Pseudomonas (52\%) was the most common isolate. In 6 children, more than 1 microorganism was isolated. Gram negatives were less common in early VAP. Eleven multiresistant Pseudomonas were detected, 7 of them were isolated during the first 3 months of the study. This lead to introduce effective control measurements for NI. Figure 3 shows pathogens isolated in VAP. Out of 16 Pseudomonas, 15 were aeruginosa and 1 fluorescens; isolated Gram positive cocci were $S$ aureus, $S$ cohnni y Streptococcus mitis (one each); one $C$ albicans and one $C$ glabrata were isolated.

\section{Figure 3. Pathogens isolated in ICU patients with VAP} UTI (7 isolates)

In $86 \%$ of cases at least one microorganism was isolated. Candida was isolated in 5/7 cases (4 albicans and $1 \mathrm{sp}$ ); One Klebsiella pneumoniae and one E coli were isolated.

All strains (5) of CNS causing BSI, were resistant to oxacillin and susceptible to vancomycin. All isolated fungi strains (except $C$ krusei) were sensible to fluconazole and voriconazole. Pseudomonas isolated in ETA had high antibiotic resistance rates: $13 / 16$ for ceftazidime, 14/16 for amikacin and ciprofloxacin and 12/16 for meropenem.

Six strains produced extended-spectrum-beta-lactamase (ESBL): one of 3 isolations of E. coli and 5 of 10 isolations of Klebsiella. Four strains were isolated from ETA (one E. coli and $3 K$. pneumoniae); two others came from blood cultures (K. oxytoca) and urine culture (K. pneumoniae). No ESBL producing strain was resistant to meropenem.

The bivariate analysis adjusted by sex, age and postoperative status found that the use of ETT (RR 22.9; CI 5.6,93.8), CVC (RR 3.6; CI 2.2,5.9) and UC (RR 2.0; CI $1.2,3.1)$ increased the risk of developing an NI. Using two devices increased the risk for NI (RR 2.6; CI

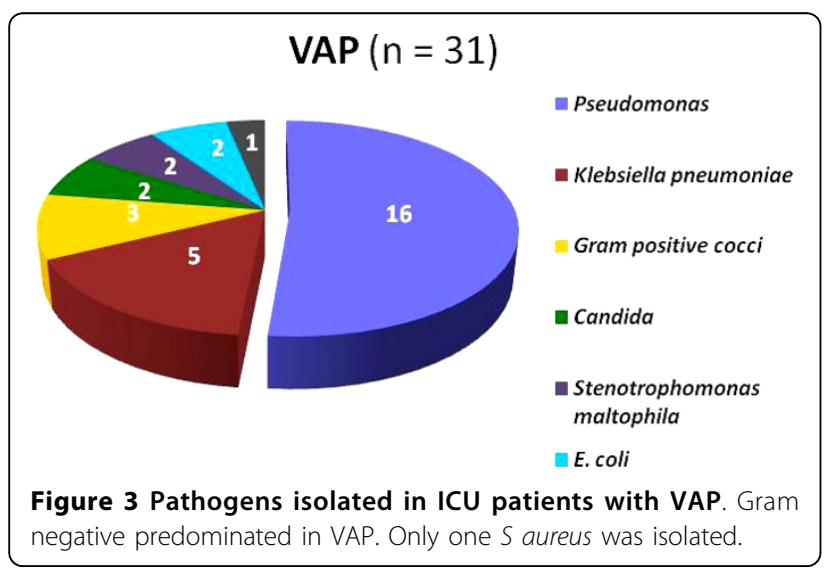

1.3,4.9), as well as using three devices (RR 3.6; CI 1.9,7.1). A 45 day stay doubled the risk for NI (RR 1.95; CI 1.6,2.3). Use of ETT for 15 days increased the risk of NI by 1.39 (CI 1.29,1.49) whilst the use of CVC and UC for 10 days increased the risk of NI by 1.73 (CI 1.52, 1.96) and 1.56 (CI 1.36,1.79) respectively.

Results of the multivariate analysis can be seen in Table 3 . It shows the 3 variables adjusted for age, sex and postoperative condition that resulted associated to NI. The presence of ETT, time of use of ETT and time of use of CVC are associated to the development of NI.

Table 3: Multivariate analysis of children in the PICU The cumulative incidence of NI is shown in Figure 4.

Figure 4. Cumulative incidence of nosocomial infections in the Pediatric Intensive Care Unit

\section{Discussion}

Surveillance of NIs may help in decreasing the incidence of infections and reducing costs. In this study, $20 \%$ of patients in a PICU of a developing country developed an NI, BSI being the most frequent. Unexpectedly, Candida spp was the most common cause of BSI.

There are some limitations of this study, particularly when it is to be compared with others [1-4,6,13]. First, we studied only 3 types of NI. Secondly, our study did not aim to identify all nosocomial pneumonias but VAPs. Despite that $95 \%$ of nosocomial pneumonias are VAPs [20], this must be borne in mind when comparing figures. Finally, we did not search for viral agents, and it is known that virus can cause almost $20 \%$ of NIs in children [21].

NIs can be defined in different ways, thus assessment and comparison can be complicated. However, NIs are better expressed using as denominator the number of patients or number of days of use of devices. Use of devices is an indicator of patient's severity of illness or of invasive routines in ICU [22] and is strongly associated to development of BSI, pneumonia and UTI [3].

As in most pediatric studies [2-4], we found BSI as the most frequent NI. Only one study in Brazil [13] and

Table 3 Multivariate analysis of children in the PICU

\begin{tabular}{lllll}
\hline & RR & $\mathbf{P}>\mathbf{z}$ & \multicolumn{1}{c}{ Cl 95\% } \\
\hline Uses ETT & & & & \\
\hline No & Ref & & & \\
\hline Yes & 14.8 & $<0.001$ & 3.6 & 61.5 \\
\hline Time of use of ETT & & & & \\
\hline Days & 1.01 & 0.024 & 1.00 & 1.02 \\
\hline Time of use CVC & & & & \\
\hline Days & 1.03 & 0.005 & 1.01 & 1.05 \\
\hline
\end{tabular}

RR: relative risk; CVC: central venous catheter; ETT: endotracheal tube. 


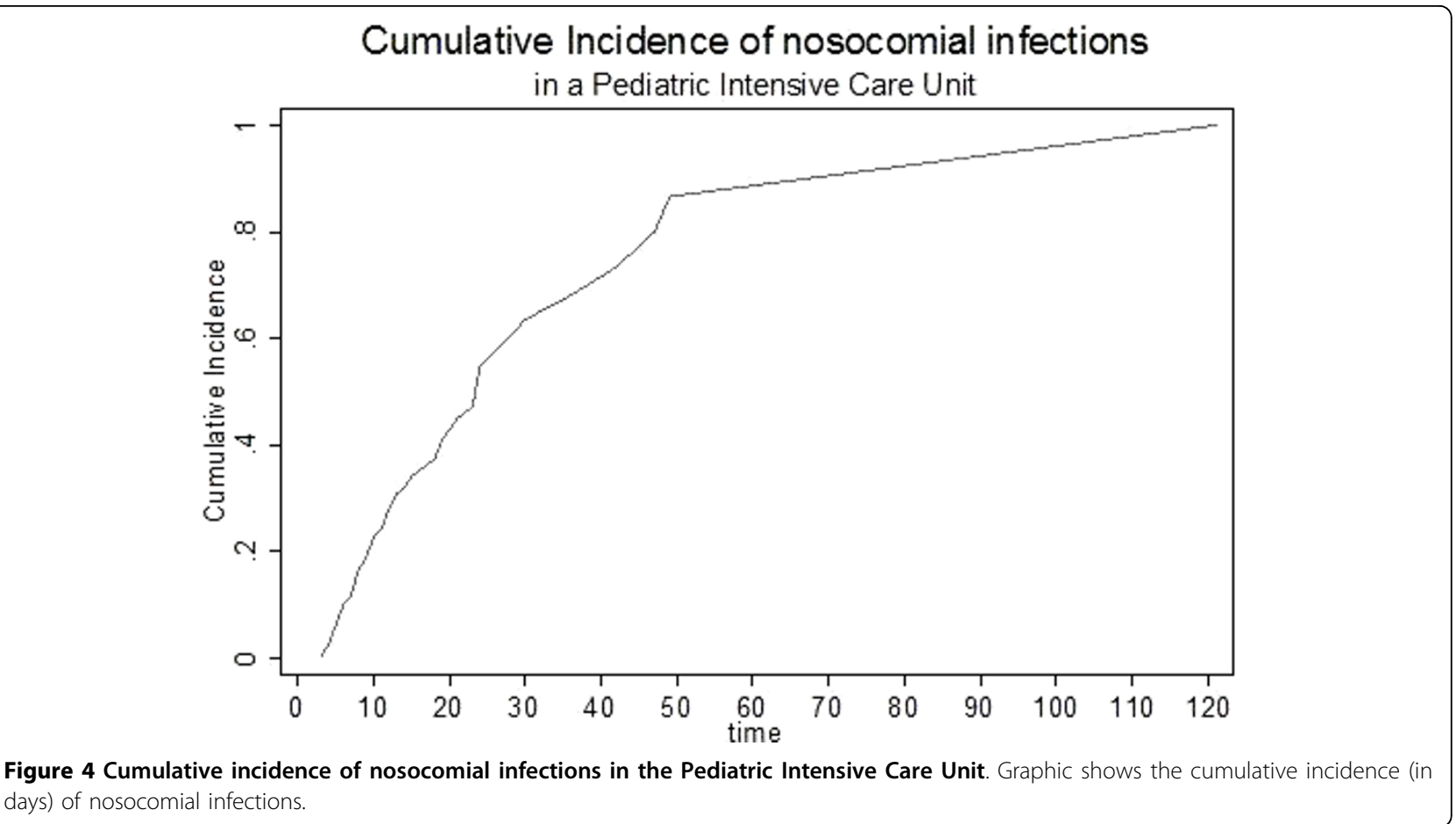

another in Europe [1] found pneumonia to be the most common.

Our ratio for use of CVC, ETT and UC (0.46, 0.74 and 0.25 respectively) was, compared with NNIS reports $(0.46,0.43$ and 0.32$)$ [22], noticeably greater for ETT and similar regarding the use of CVC and UC. Despite similar use of CVC we had more BSI (18.1 vs. 7.3 - 8.9 per 1000 days of use of CVC $[3,22])$; this fact has also been described for NI in adult ICUs in developing countries [15]. It is likely that this increase in frequency of BSI is due to a greater susceptibility of our patients, differences in catheter insertion techniques or further care, or both. The aforementioned study [15] attributed the greater frequency of NI to less frequency of hand hygiene, low nurse-to-patient-ratios, and outdated technology.

The density of incidence for VAP and UTI seem to correspond to greater and similar use of ETT and UC, respectively. On the other hand, two studies regarding risk factors found $13.8 \mathrm{BSI} / 1000$ days of use of CVC [23] and 11.6 VAP/1000 days of use of ETT [9], whilst a surveillance study for VAP found 8.9 VAP/1000 days of use of ETT [12]; these figures are closer to ours. Increased VAP rates could be due to greater rate of device use [22], as mentioned previously, but also to other factors. In our study, $30 \%$ of patients had a genetic syndrome, and the use of antibiotics (90\%), sedation (75\%), transfusions (70\%), $\mathrm{H}_{2}$ blockers (60\%), steroids $(50 \%)$ and inotropes $(40 \%)$ was highly prevalent; all these have been considered as potential risk factors for VAP $[9,12]$.

The isolation of Candida spp. as the most common isolate in our children with BSI, in contrast with studies that show CNS as the principal causing agent [2-5,23,24], has already been pointed out [4]. There has been an increase in Candida BSI infections over the last years, as well as a shift toward non-albicans Candida $[24,25]$. Risk factors for candidemia are previous colonization, long ICU stays, presence of CVC, parenteral nutrition, $\mathrm{MV}$, illness severity and prolonged use of antibiotics [25-27]. It is likely that the most important cause of our high prevalence of Candida is the extensive use of broad spectrum antibiotics [26]. Although at INSN vancomycin, carbapenems and ciprofloxacin must be approved by an infectologist before its use, third generation cephalosporins, vancomycin, and amikacin are frequently used at our institution, and more than $90 \%$ of PICU patients received any of these antibiotics or ciprofloxacin both in the PICU and previous to PICU admission. Long ICU stay is also described as a risk factor for candidemia [26], and although not described in the results section, children with BSI due to Candida had ICU stay almost twice longer than children with BSI due to other microorganisms (average, 53 vs. 27 days). Also not shown, previous surgery in children under 12 months was more frequently seen in children with BSI due to Candida than in children with BSI due to other microorganisms (75\% vs. $29 \%$ ). We have no 
explanation for this finding, but maybe it could be researched in future investigations. Other possible causes are previous colonization with Candida, due to the high use of antibiotics, as well as our high use of intubation and MV [26]. Candidemia is particularly important, because it is associated with high mortality, both in adults [28] and children, especially at lower ages and in those with comorbidities [26]. Due to its increasing numbers, more studies about risk factors for Candida infection are needed.

Gram negatives, mainly Pseudomonas, caused $80 \%$ of VAP in our study. $P$ aeruginosa is the most common organism in children with VAP, followed by other Gram negatives and $S$. aureus $[1,3,4,9,12,29,30]$. There was just one case of $S$. aureus in our study, possibly because many children are admitted to the ICU having been treated with vancomicin.

The most common organisms in UTI are E. coli $[2,3]$ or, as in our study, Candida $[4,31]$. We found 5 Candida, 4 of them were associated to urinary catheters.

We had a high rate of antibiotic resistance. All CNS causing BSI were resistant to oxacillin and around $80 \%$ of Pseudomonas in children with VAP were resistant to ceftazidime, amikacin, ciprofloxacin and meropenem. Resistance pattern is high at INSN: $50 \%$ of $S$ aureus are resistant to oxacillin, $85 \%$ of Klebsiella are resistant to third generation cephalosporin and $50-90 \%$ of $P$ aeruginosa are resistant to carbapenems, amikacin, ceftazidime and ciprofloxacin (source: Committee on Nosocomial Infections). We believe that this is due to the extensive use of antibiotics.

Mortality of NI in the present study was $38 \%$, notably greater than that reported from PICUs of developed countries $(7.7-10 \%)[1,2]$, and somewhat greater than that from a PICU of Brazil (28.3\%) [13]. Mortality in Latin American countries tends to be greater than that in the USA. A study in 6 PICUs from this region found greater mortality than expected among lower risk patients (PRISM < 10), compared to one PICU in USA, which could be explained on the basis of increased use of central lines and ETT as well as broad spectrum antibiotics [32].

Described mortality for BSI is $14-26.5 \%$ [5,23,24]; we found $31 \%$, this could be related to greater severity of disease, differences in management or both. Notwithstanding we found greater mortality (46\%) for BSI due to Candida than with other organisms, the limited number does not allow us comparative analysis.

Nosocomial pneumonia is the main cause of death in children with NI [30], but it is doubtful if VAP increases mortality in children on MV. In contrast to other studies $[9,12]$, our mortality for VAP was significantly greater than for children without VAP. In a study from a developing country, the risk adjusted PICU mortality was significantly greater in VAP versus non-VAP patients [33]. Mortality for VAP seems also be related to the causative pathogen and the time of onset $[8,19]$. For example, Gram negative bacilli (particularly Pseudomonas) have $>80 \%$ mortality [8], and early VAP has smaller mortality than late VAP [19]. Our limited numbers (5 early and 22 late VAPs) forbid further analysis, but we found a tendency to greater mortality in late VAP. We have found no pediatric studies that have researched on this topic beyond the neonatal area.

A striking feature of our data is that ICU stay previous to NI diagnosis was greater for this group than for children who did not develop an infection. It is known that NIs increase hospital stay $[1,9,12,23]$, but also a longer stay per se is a risk factor for NI [34]. That suggests that patients who developed an NI were already significantly different from those who did not, which is particularly important for future studies and when interpreting the impact of the NI. Since a significant portion of our patients had co-morbidity, it is possible that its condition resulted in a longer stay, predisposing them to NI. By the other hand, although in bivariate analysis ICU stay was associated to NI development, in multivariate analysis it did not result significant.

This study has several restrictions, like the lack of inclusion of data on re-intubation, which could be relevant for VAP; likewise, the sample collecting technique (tracheal aspiration) is not the one recommended by most authors. PRISM score was recorded in only $22 \%$ of patients. Neither did we stratify cases according to severity of disease, which could influence the appearance of NI, mortality and stay. Finally, it must be borne in mind that the INSN is a national reference center and admits children from other institutions, thus our results may differ from other PICUs. Additionally, we were not able to calculate attributable mortality because data that could have influenced the results was not available.

\section{Conclusions}

This study highlights the importance of NIs in children admitted to a PICU in a developing country. Patients in these units, despite representing a small percentage of inpatients, contribute with over $20 \%$ of NIs [35]. Potential impact of NI control is such that of 6 recommendations to improve interventions in health, issued by the Institute for Health Improvement, 3 are directly related to actions to decrease NIs [20]. In the ICU at the INSN one of every 5 children acquires an NI, which was associated with increased mortality and length of stay.

\section{Acknowledgements}

We thank the PICU staff of the Instituto Nacional de Salud del Niño and Instituto Nacional de Salud for their collaboration with this study, as well as 
those from the Study Group of Nosocomial Infections in Peru: Justo $R$ Padilla, MD, Felipe R Lindo, MD, Lilian L Patiño, MD, Cecilia J Taboada, MD, Mirian M Guevara, Blg, Rosa E Sacsaquispe, Blg.

Financial support: This study was supported by the Instituto Nacional de Salud del Niño and the Instituto Nacional de Salud of Lima, Peru. The authors state that they do not have conflict of interest about this article.

\section{Author details}

'Pediatric Intensivist, Instituto Nacional de Salud del Niño, Lima, Peru. ${ }^{2}$ Pediatric Intensivist, Master in Medicine, Instituto Nacional de Salud del Niño and Universidad Nacional Federico Villarreal, Lima, Peru. ${ }^{3}$ Infectologist, Tropical Infectious Diseases, Instituto Nacional de Salud, and Universidad César Vallejo, Lima, Peru. ${ }^{4}$ Nurse, Instituto Nacional de Salud del Niño, Lima, Peru. ${ }^{5}$ Pediatric Infectologist, Instituto Nacional de Salud del Niño, and Universidad San Martín de Porres, Lima, Peru. ${ }^{6}$ Biologist, Instituto Nacional de Salud, Lima, Peru.

\section{Authors' contributions}

MRB made substantial contributions to acquisition, analysis and interpretation of data, was involved in drafting the manuscript and approved its final version. JAT conceived the study, participated in design, analysis and interpretation of data, was involved in drafting the manuscript and approved its final version. VJS made substantial contributions to design, analysis and interpretation of data, was involved in drafting the manuscript and approved its final version. MCA made substantial contributions to acquisition and interpretation of data and approved its final version. JLC made substantial contributions to analysis and interpretation of data, was involved in drafting the manuscript and approved its final version. FCU made substantial contributions to acquisition and interpretation of data and approved its final version

\section{Competing interests}

The authors declare that they have no competing interests.

Received: 6 January 2010 Accepted: 10 September 2010 Published: 10 September 2010

\section{References}

1. Raymond J, Aujard Y, Bekassi A, the European Study Group: Nosocomial infections in pediatric patients: a European, multicenter prospective study. Infect Control Hosp Epidemiol 2000, 21:260-263.

2. Urrea M, Pons M, Serra M, Latorre C, Palomeque A: Prospective incidence study of nosocomial infections in a pediatric intensive care unit. Pediatr Infect Dis J 2003, 22:490-493.

3. Richards MJ, Edwards JR, Culver DH, Gaynes R: Nosocomial infections in pediatric intensive care units in the United States. Pediatrics 1999, 103: e39.

4. Grohskpof L, Sinkowitz-Cochran R, Garrett D, Sohn A, Levine G, Siegel J, Stover B, Jarvis W, the Pediatric Prevention Network: A national pointprevalence survey of pediatric intensive care unit-acquired infections in the United States. J Pediatr 2002, 140:432-438,

5. Gray J, Gossain S, Morris K: Three-year survey of bacteremia and fungemia in a pediatric intensive care unit. Pediatr Infect Dis J 2001, 20:416-421.

6. Stover B, Shulman S, Brathcer D: Nosocomial infection rates in US children's hospital neonatal and pediatric intensive care units. Am J Infect Control 2001, 29:152-157.

7. Huskins Ch, Goldmann D: Nosocomial infections. In Textbook of Pediatric Infectious Diseases. Edited by: Feigin, Cherry, Demmler, Kaplan. Saunders; , 5 2004:2874-2925.

8. Chastre J, Fagon JY: Ventilator - associated Pneumonia. Am J Respir Crit Care Med 2002, 165:867-903.

9. Elward A, Warren D, Fraser V: Ventilator- associated pneumonia in pediatric intensive care unit patients: Risk factors and outcomes. Pediatrics 2002, 109:758-764.

10. Rivera $\mathrm{R}$, Tiballs J: Complications of endotracheal intubation and mechanical ventilation in infants and children. Crit Care Med 1992, 20:193-199.
11. Barzilay Z, Mandel M, Keren G, Davidson S: Nosocomial bacterial pneumonia in ventilated children: Clinical significance of culture-positive peripheral bronchial aspirates. J Pediatrics 1988, 112:421-424.

12. Almuneef $M$, Memish $Z$, Balkhy $H$, Alalem $H$, Abutaleb A: VentilatorAssociated Pneumonia in a Pediatric Intensive Care Unit in Saudi Arabia: A 30 month prospective surveillance. Infect Control Hosp Epidemiol 2004, 25:753-758.

13. Abramczyk M, Carvalho W, Carvalho E, Medeiros E: Nosocomial Infection in a Pediatric Intensive Care Unit in a Developing Country. Braz J Infect Dis 2003, 7(6):375-380

14. Tantaleán J, León R, Santos A, Sánchez E: Multiple organ dysfunction syndrome in children. Pediatr Crit Care Med 2003, 4:181-185.

15. Rosenthal V, Maki D, Salomao R, Alvarez-Moreno C, Mehta Y, Higuera F, Cuellar L, Arikan O, Abouqal R, Lebleblcloglu $\mathrm{H}$, for the International Nosocomial Infection Control Consortium: Device-associated nosocomial infections in 55 Intensive Care Units of 8 developing countries. Ann Intern Med 2006, 145:582-591.

16. Garner JS, Jarvis WR, Emori TG, Horan TC, Hughes JM: CDC definitions for nosocomial infections. In APIC Infection Control and Applied Epidemiology: Principles and Practice. Edited by: Olmsted RN. St. Louis: Mosby; 1996:A-1-A20.

17. Goldstein B, Giroir B, Randolph A: International Pediatric Sepsis Consensus Conference: Definitions for sepsis and organ dysfunction in pediatrics. Pediatr Crit Care Med 2005, 6:2-8.

18. See LL: Bloodstream infection in children. Pediatr Crit Care Med 2005, 6(3 Suppl):S42-S44.

19. American Thoracic Society Documents: Guidelines for the management of adults with Hospital-acquired, Ventilator-associated, and Health-care associated Pneumonia. Am J Respir Crit Care Med 2005, 171:388-416.

20. Stockwell JA: Nosocomial infections in the pediatric intensive care unit: Affecting the impact on safety and outcome. Pediatric Crit Care Med 2007, 8(suppl):S21-S37.

21. Harris J: Pediatric nosocomial infections: children are not little adults. Infect Control Hosp Epidem 1997, 18(11):739-742.

22. National Nosocomial Infections Surveillance (NNIS) System: National Nosocomial Infections Surveillance (NNIS) System Report, data summary from January 1992 through June 2003, issued August 2003. Am J Infect Control 2003, 31:481-498.

23. Yogaraj J, Elward A, Fraser V: Rates, risk factors and outcomes of nosocomial primary bloodstream infection in Pediatric Intensive Care Unit patients. Pediatrics 2002, 110:481-485.

24. Wisplinghoff $H$, Seifert $H$, Tallent $S$ : Nosocomial bloodstream infections in pediatric patients in United States hospitals: epidemiology, clinical features and susceptibilities. Pediatr Infect Dis J 2002, 22:686-691.

25. Singhi S, Rao R, Chakrabarti A: Candida colonization and candidemia in a pediatric intensive care unit. Pediatr Crit Care Med 2008, 9:91-95.

26. Marodi $L$, Johnston R: Invasive Candida species disease in infants and children: occurrence, risk factors, management, and innate host defense mechanisms. Curr Opin Pediatr 2007, 19:693-697.

27. García L, Cobo J, Martos I, Otheo E, Muriel A, Pintado V, Moreno S: Risk Factors for Candidemia in Pediatric Patients With Congenital Heart Disease. Infect Control Hosp Epidemiol 2006, 27:576-580

28. Chow J, Golan Y, Ruthazer R: Risk factors for albicans and non-albicans candidemia in the intensive care unit. Crit Care Med 2008, 36:1993-1998.

29. Foglia E, Meier M, Elward A: Ventilator-Associated Pneumonia in Neonatal and Pediatric Intensive Care Unit Patients. Clinical Microbiology Reviews 2007, 409-425.

30. Zar H, Cotton M: Nosocomial Pneumonia in Pediatric Patients. Practical Problems and Rational Solutions. Pediatr Drugs 2002, 4(2):73-83.

31. Matlow A, Wray R, Cox P: Nosocomial urinary tract infections in children in a pediatric intensive care unit: A follow-up after 10 years. Pediatr Crit Care Med 2003, 4:74-77.

32. Earle M Jr, Martinez O, Zaslavsky A, Quiñones E, Carrillo H, García E, Zavala I, García R, Todres I: Outcome of pediatric intensive care at six centers in Mexico and Ecuador. Crit Care Med 1997, 25(9):1462-7.

33. Morrow $B$, Argent A: Ventilator-associated pneumonia in a paediatric intensive care unit in a developing country with high HIV prevalence. $J$ Paediatr Child Health 2009, 45(3):104-11. 
34. Gilio A, Stape A, Pereira C, Cardoso M, Silva C, Troster E: Risk factors for nosocomial infections in a critically ill pediatric population: A 25-month prospective cohort study. Infect Control Hosp Epidemiol 2000, 21:340-342.

35. Siegel JD, Rhinehart E, Jackson $M$, Chiarello $L$, the Healthcare Infection Control Practices Advisory Committee: 2007 Guideline for Isolation Precautions: Preventing Transmission of Infectious Agents in Healthcare Settings. 2008 [http://www.cdc.gov/ncidod/dhqp/pdf/guidelines/ isolation2007.pdf].

Pre-publication history

The pre-publication history for this paper can be accessed here: http://www.biomedcentral.com/1471-2431/10/66/prepub

doi:10.1186/1471-2431-10-66

Cite this article as: Becerra et al:: Epidemiologic surveillance of

nosocomial infections in a Pediatric Intensive Care Unit of a developing country. BMC Pediatrics 2010 10:66

Submit your next manuscript to BioMed Central and take full advantage of:

- Convenient online submission

- Thorough peer review

- No space constraints or color figure charges

- Immediate publication on acceptance

- Inclusion in PubMed, CAS, Scopus and Google Scholar

- Research which is freely available for redistribution

Submit your manuscript at www.biomedcentral.com/submit 